\title{
Seminal vesicle abscess causing unilateral hydroureteronephrosis: A case report
}

\author{
Vittorio Imperatore ${ }^{1}$, Massimiliano Creta ${ }^{1}$, Sergio Di Meo ${ }^{1}$, Roberto Buonopane ${ }^{1}$, \\ Lorenzo Spirito ${ }^{2}$, Vincenzo Mirone ${ }^{2}$ \\ ${ }^{1}$ Operative Unit of Urology, Buon Consiglio Hospital - Fatebenefratelli, Naples, Italy; \\ ${ }^{2}$ Urological Clinic, Federico II University of Naples, Naples, Italy.
}

\begin{abstract}
Summary Seminal vesicle abscess (SVA) is a rare urologic entity. It mainly occurs in subjects with predisposing factors and may be associated with other urogenital infections. We describe the case of a diabetic subject with SVA associated with funiculitis, epididymitis and obstructive pyelonephritis. Treatment consisted of laparotomic surgical drainage of the abscess and ureteral stent placement.
\end{abstract}

KEY WORDS: Diabetes; Hydroureteronephrosis; Seminal Vesicle Abscess.

Submitted 15 August 2017; Accepted 21 September 2017

\begin{abstract}
INTRODUCTION
Currently, seminal vesicle abscess (SVA) is rarely encountered in everyday clinical practice mainly due to the widespread use of broad spectrum antibiotics in subjects with urinary tract infections $(1,2)$. However, it may occur in the presence of predisposing conditions such as: anatomic abnormalities, compromised immunity, diabetes, prolonged catheterization, urinary tract infections and instrumentation $(1,2)$. Moreover, SVA frequently occurs in concomitance with other urogenital infections such as acute prostatitis, prostate abscess, epididymitis, and funiculitis. Clinical diagnosis of SVA is often challenging due to nonspecific clinical symptoms $(1,2)$. Treatment modalities for SVA include conservative management with long-term antimicrobial therapy or drainage $(1,2)$. SVA drainage can be achieved via laparotomic, percutaneous, transrectal or transurethral accesses. However, due to the rarity of the disease, there are no specific guidelines for the management of this urological entity. We describe the case and clinical management of a diabetic patient presenting with a large SVA associated with funiculitis, epididymitis, and unilateral obstructive acute pyelonephritis.
\end{abstract}

\section{Case report}

A 58-year-old man presented to the emergency department with a 7-day history of dysuria and fever. His past medical history was relevant for diabetes mellitus with irregular medical control. A complete blood count demonstrated white blood cells of $23.94 \times 10^{\wedge} 3 / \mathrm{ml}$ with elevated neutrophil count (89.3\%). Serum urea nitrogen and creatinine concentrations were $106 \mathrm{mg} / \mathrm{dl}$ and 1.73 $\mathrm{mg} / \mathrm{dl}$, respectively. Blood glucose level was $527 \mathrm{mg} / \mathrm{dl}$ and $\mathrm{HbAlC}$ was $16.7 \%$. Prostate specific antigen level was $0.7 \mathrm{ng} / \mathrm{mL}$. Urinalysis showed pyuria, glycosuria and ketonuria. On digital rectal examination, the prostate could not be easily palpated and a bulging of the rectal wall was evident cranially to the prostate. The left spermatic cord and hemiscrotum were swollen. Findings from scrotal ultrasound were compatible with left funiculitis associated with epididymitis and the abdominal ultrasound revealed a right hydroureteronephrosis. Intravenous empirical broad-spectrum antibiotic treatment with piperacillin/tazobactam was immediately started. A Computed tomography (CT) of the abdomen and pelvis revealed the presence of a $6 \times 5 \mathrm{~cm}$, multiloculate pelvic abscess involving the recto-prostatic and the recto-vesical spaces (Figure 1).

The abscess was adjacent to the bladder and to the rectosigmoid colon that appeared irregularly thickened. Moreover, a right hydroureteronephrosis and ipsilateral pyelonephritis were evident. A colonoscopy and a watersoluble contrast enema were required. Colonoscopy confirmed an irregular thickening of the rectosigmoid junction and excluded malignancy. The enema excluded the presence of fistulous tracts. A SVA was suspected based on clinical, biochemical and radiological findings.

\section{Figure 1.}

Transverse (A, D), sagittal (B) and coronal

(C) computed tomography sections of the pelvis showing a $6 \times 5 \mathrm{~cm}$, multiloculate abscess (*) involving the recto-vesical and the recto-prostatic spaces. Bladder (b) and rectum ( $r$ ) walls were irregularly thickened. Right ureteral dilatation was evident (arrow).

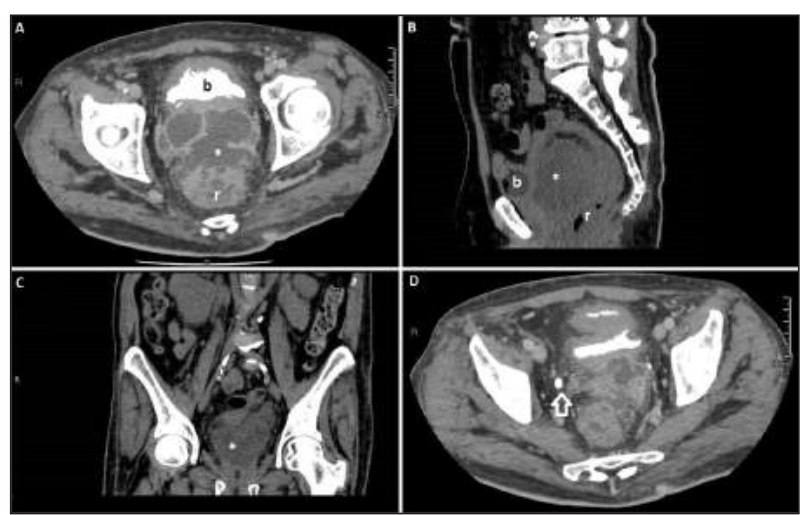


Due to the inadequate clinical response to conservative treatment, an open surgical drainage was planned via a median sub umbilical laparotomy. An extraperitoneal approach failed to reach the abscess. Consequently, the peritoneum was opened and the abscess was reached through the pouch of Douglas. The abscess was drained, the seminal vesicles appeared necrotic so they were removed and sent for histological examination. No other pathological findings were evident. A drainage was left in situ for 5 days. A right ureteral stent was endoscopically positioned. There were no intraoperative or post-operative complications.

The operative time was 90 minutes. The postoperative course was uneventful and the patient was discharged home on post-operative day 7 . Histological examination revealed necrotic tissues.

\section{Discussion}

SVA represents a rare pathological entity with only few cases reported in the international literature $(1,2)$. The anatomical relationship of seminal vesicles with neighboring organs such as prostate, bladder, ureter, rectum, and peritoneum is responsible for the wide variety of presenting symptoms, the complex diagnostic work-up and the challenging surgical therapy. SVA may occur in isolation or, more frequently, in association with other genitourinary infections such as prostatitis, prostate abscess, funiculitis, or epididymitis $(1,2)$. The symptoms of SVA are nonspecific and may include fever, chills, irritative lower urinary tract symptoms, hematuria, scrotal, perineal, or rectal pain $(1,2)$. On physical examination, a mass can be palpated above the prostate that can be normal. However, due to the anatomic location, SVA cannot be palpated in some cases (2). Therefore, the diagnosis requires a high index of clinical suspicion. To our knowledge, we have described the first case of SVA causing unilateral ureteral obstruction and pyelonephritis. Prostate abscesses, cysts and neoplasms should be included into the differential diagnosis $(1,2)$. Transrectal prostate ultrasound, CT and magnetic resonance are useful imaging modalities in patients with pelvic masses and abscess (1-3). However, CT remains the most commonly prescribed diagnostic tool in cases of SVA. It allows to identify predisposing factors and to define the borders of the abscess. Typical CT findings include: unilateral or bilateral seminal vesicle enlargement with a central irregular low-density zone, inflammation of the surrounding fat, thickening of the adjacent organs $(1,2)$. Although prostate specific antigen level was within the normal range, a concomitant prostate abscess could not be excluded in the present case. If left untreated, both prostate and SVA abscesses may burst into perirectal tissues, the perineum, form a fistula, and are associated with a risk of mortality. Conservative medical treatment with antibiotics has been reported to be efficacious in selected cases $(1,2)$. Necrotizing complications may potentially occur. As in other biological fields, necrotic tissues may act as inert bodies thus favoring the persistence of infection (4). Some patients may not respond to conservative treatment and abscess drainage is required. Multiple drainage techniques have been reported such as transperineal, transgluteal, or transrectal need aspiration, transurethral incision, and surgical drainage via laparotomy. Both the trans-abdominal and the trans-perineal routes can be utilized to access to the recto-vesical and the recto-prostatic spaces (5). When surgical drainage is planned, image findings allow to choose the more appropriate approach. In cases of multiloculate abscesses, needle drainage may require multiple punctures and a high risk of persistence has been reported. In the present case, we planned the drainage of the abscess due to the extent of the collection and to the unsatisfactory response to conservative medical therapy. A laparotomic approach was adopted due to the multiloculate anatomy of the abscess and its localization that made it not easily reachable via the trans-perineal route.

\section{Conclusions}

SVA is a rare urological entity that should be suspected in subjects with evidence of pelvic abscesses and predisposing factors. It may occur in the contest of multiple genitourinary infections and may be responsible for upper urinary tract obstruction. Open surgical drainage can be required in selected cases.

\section{REFERENCES}

1. Kang YS, Fishman EK, Kuhlman JE, Goldman SM. Seminal vesicle abscesses: spectrum of computed tomographic findings. Urol Radiol. 1989; 11:182-5.

2. Saglam M, Ugurel S, Kilciler M, et al. Transrectal ultrasoundguided transperineal and transrectal management of seminal vesicleabscesses. Eur J Radiol. 2004; 52:329-334.

3. Creta M, Mirone V, Di Meo S, et al. A rare case of male pelvic squamous cell carcinoma of unknown primary origin presenting as perineal abscess and urethral stenosis. Arch Ital Urol Androl. 2017; 89:154-155

4. Cennamo P, Montuori N, Trojsi G, et al. Biofilms in churches built in grottoes. 2016; 543:727-38.

5. Imperatore V, Creta M, Di Meo S, et al. Transperineal repair of a persistent rectourethral fistula using a porcine dermal graft. Int $J$ Surg Case Rep. 2014; 5:800-2.

\section{Correspondence}

Vittorio Imperatore, MD (Corresponding Author)

v.imperatore@alice.it

Massimiliano Creta, MD

max.creta@gmail.com

Sergio Di Meo, MD

s.dimeo72@gmail.com

Roberto Buonopane, MD

robertobuonopane@libero.it

Operative Unit of Urology, Buon Consiglio Hospital - Fatebenefratelli, Naples, Italy

Via A. Manzoni, 220, 80123 Napoli

Lorenzo Spirito, MD

Vincenzo Mirone, MD

Urological Clinic, Federico II University of Naples, Naples, Italy

Via S.Pansini, 5, 80131 Napoli 